\title{
GIS BASED SYSTEM FOR POST-EARTHQUAKE CRISIS MANAGMENT USING CELLULAR NETWORK
}

\author{
M. Raeesi ${ }^{\text {a }}$, A. Sadeghi-Niaraki ${ }^{\text {a }}$ \\ ${ }^{a}$ GIS Dept. Faculty of Geodesy and Geomatics Eng, K.N.Toosi Univ. of Tech., Tehran, Iran - \\ moein_3030@yahoo.com, a.sadeghi@kntu.ac.ir
}

KEY WORDS: GIS, cellular network, post-earthquake crisis management

\begin{abstract}
:
Earthquakes are among the most destructive natural disasters. Earthquakes happen mainly near the edges of tectonic plates, but they may happen just about anywhere. Earthquakes cannot be predicted. Quick response after disasters, like earthquake, decreases loss of life and costs. Massive earthquakes often cause structures to collapse, trapping victims under dense rubble for long periods of time. After the earthquake and destroyed some areas, several teams are sent to find the location of the destroyed areas. The search and rescue phase usually is maintained for many days. Time reduction for surviving people is very important. A Geographical Information System (GIS) can be used for decreasing response time and management in critical situations. Position estimation in short period of time time is important. This paper proposes a GIS based system for post-earthquake disaster management solution. This system relies on several mobile positioning methods such as cell-ID and TA method, signal strength method, angel of arrival method, time of arrival method and time difference of arrival method. For quick positioning, the system can be helped by any person who has a mobile device. After positioning and specifying the critical points, the points are sent to a central site for managing the procedure of quick response for helping. This solution establishes a quick way to manage the post-earthquake crisis.
\end{abstract}

\section{INTRODUCTION}

\subsection{Motivation}

One of the main elements for reducing response time is the number of rescuers. The number of rescuers to help people who need help is restricted. So a system is defined that should provide a mechanism for obtaining a quick information from public people (public participation). The location estimation of a MS (mobile subscriber) in cellular network provides many services. We want to use this positioning to reduce the response time for post-earthquake.

\subsection{Aims}

After the disaster, each citizen who has a mobile device with calling or messaging to a certain number (specified for positioning), the location of critical points can be specified. The goal is to design and introduce the architecture of a system to reduce the time of rescue operations with the help of public participation.

\subsection{Overview and references to related work}

Most previous works have focused on methods of mobile positioning, combining different methods and taxonomies of location determination techniques. An article described advances of positioning methods in cellular networks (Samiei, Mehrjoo, Pirzade, 2010). This paper has illustrated several methods for positioning of MS based on cellular network and in the next section comparison between methods based on accuracy, cost and applicability. The authors in (Samiei, Mehrjoo, Pirzade, 2010) suggest applying signal attenuation as weights in the cost function of the time-based methods to improve the measurement accuracy. In the hybrid method which has been proposed in (G. Kbar, W. Mansoor, 2005), the signal strength has been used to estimate the timing delay which is compared with the measured time of arrival. The average of the minimum timing value of the two results can then be used in measurement. A new signal strength-based method, Signal Attenuation Difference of Arrival (SADOA) method is combined with TDOA method in (Juang, Lin, 2006). SADOA is based on the ratio of distances between the mobile and base stations derived from differences of signal attenuations. Authors in (Wan Bejuri, Wan Mohd. Yaakob1, Mohamad, Mohd. Murtadha1 and Sapri, 2011) state that there are many different positioning methods and classified this methods based on scale, output, measurement, rules and estimation method, in three sections. Three sections are radio frequency-based method, camera navigation-based method and GPS-based method. This article is useful for deciding each method where should be used. The authors in (Doukhnitch, Salamah, 2008) propose a general approach to design new hardware-oriented algorithms that use only simple add and shift operations in the computation and therefore can be easily implemented in hardware or firmware. In addition to their implementation simplicity, the proposed algorithms outperform the conventional ones in terms of the required number of operations for a specific accuracy level.

\section{METHODOLOGY}

The methodology of the research includes two parts. First part is a mobile positioning with BTS (Base Transceiver Station) antenna and second part is specifying the range of service and road finding analysis. In the first part with the help of positioning system after natural disasters, people specify the 
critical points that need to aid. For specifying this critical point, a mobile device can be used. A positioning method for specifying the location of the mobile device is needed. For mobile positioning, several methods assuming the infrastructure is a cellular network can be used such as Cell-ID and TA method, signal strength method, angel of arrival method and time of arrival method. The mobile positioning in cellular network with the help of BTS (Base Transceiver System) antenna is used because of the each mobile device that has not GPS service can specify critical point too. For this research signal strength method is used for positioning. In this method, if three or more BTS antenna are available for receiving signal strength in cellular network, with the help of propagation models, the distance between MS (Mobile Subscriber) and BTS can be calculated. With intersection formula and at least three distances, the position of MS can be specified. The second part contains two sections. In the section one, representing critical points and specifying the range of service for rescue stations and road finding are main steps. For the second section, with this system after disaster a number of critical points are stored and location of critical points and rescue stations can be represent on a map with a GIS Software. With specifying the range of service for each rescue station, critical points belong to the specific rescue station. Routings between the critical point and related rescue station can be done. So the path of critical points related to each station is determined and can be used for rescuers navigation to critical points. Fig. 1 shows methodology flowchart for this research.

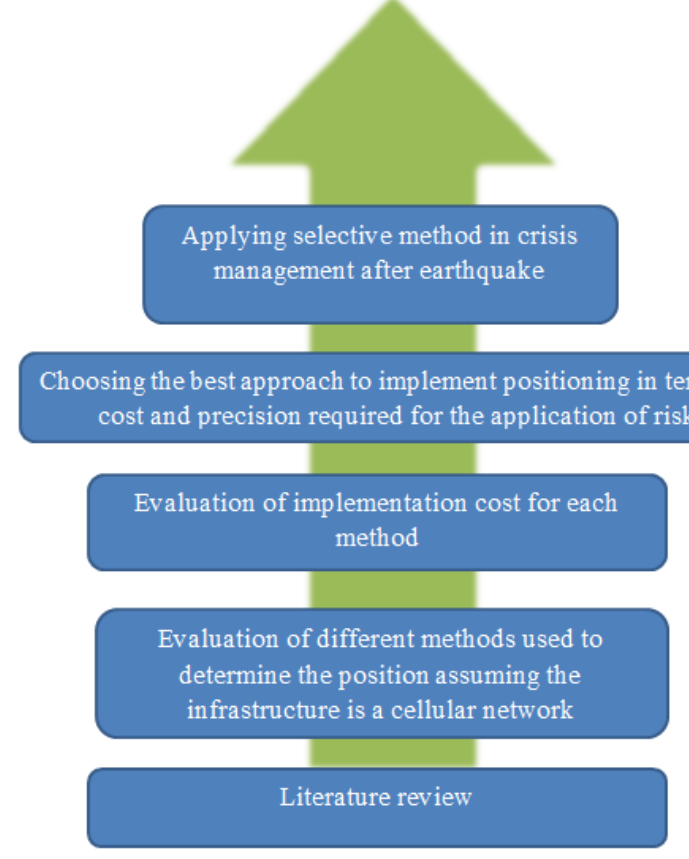

Figure 1. Methodology flowchart

\subsection{Cell-ID and TA Method}

Positioning in this method can be estimated by using the identification codes assigned to each active (communicating) MS. In this method, there is a unique specific code for each cell such as Mobile Country Code (MCC), Mobile Network Code (MNC), Location Area Code (LAC) and Cell Identity (CI). This method has a low accuracy and it is directly depend on the cell size which varies from 100 meter in urban areas to 1500 meter in rural areas (Samiei, Mehrjoo, Pirzade, 2010). Difference in accuracy between the two areas is due to vary in cell size. Positioning accuracy can be reduced by using Time Advance (TA) which is a measure of distance between MS and the Base Transceiver Station (BTS). TA is a number from 0 to 63 (Samiei, Mehrjoo, Pirzade, 2010), and one increment of TA corresponds to being approximately $554 \mathrm{~m}$ away from BTS (Samiei, Mehrjoo, Pirzade, 2010), as shown in Fig. 2. So if this number specified for MS, approximate position can be estimated with the help of sector ID. Sector ID together TA makes position narrow down to a ring of 554 meter in the identified sector, as shown as in Fig. 2.

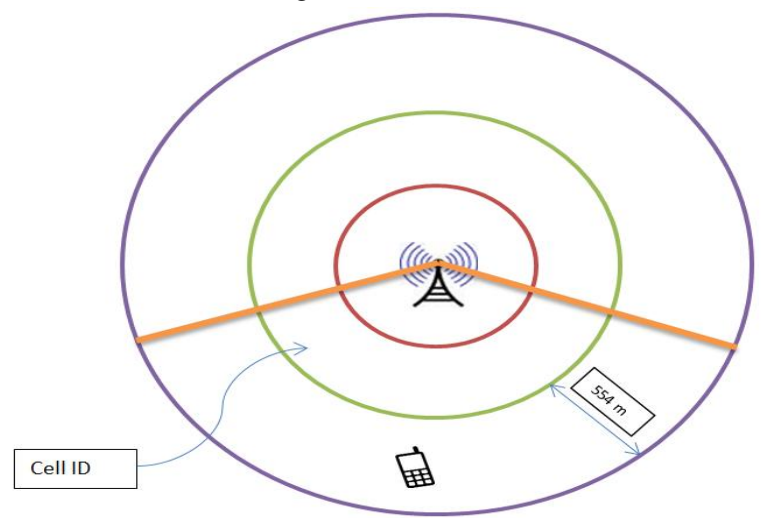

Figure 2. Cell ID \& TA positioning method

\subsection{Signal Strength Method (SSM)}

If three or more distances between MS and several BTS are measured, with the help of least square method and intersection formula the approximate position of MS can be determined. The distance between BTS and MS are calculated by propagation model (equation (1)). In this method, by measuring signal strength, distance between BTS and MS and finally position estimation will be calculated.

$$
P_{E}=P_{S}\left(\frac{\lambda}{4 \pi d}\right) g_{r} g_{t}
$$

Where: $P_{E}$ is the power of initial signal is sent to BTS, $P_{S}$ is the power of received signal in antenna, $d$ is distance between BTS and MS, $g_{r}$ and $g_{t}$ are gain of receiver and transceiver and $\lambda$ is the wave length.

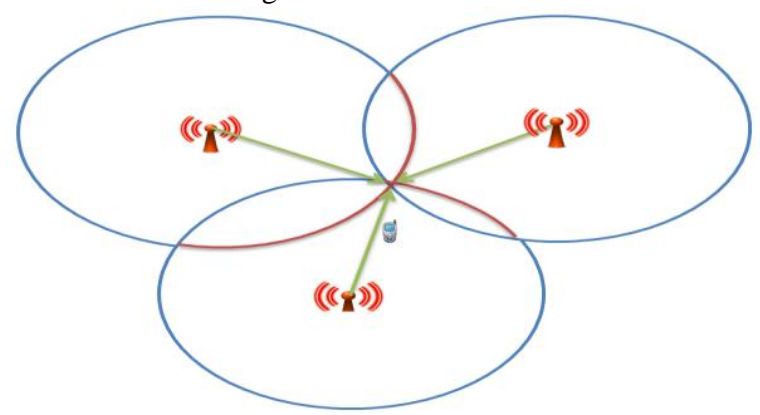

Figure 3. Signal Strength Positioning Method 


\subsection{Angle of Arrival Method (AOA)}

This method involves measuring the vertical angle of arrival of a signal from a BTS at a MS or angle of arrival of a signal from a MS at a BTS. Angle measurement provides a virtual direct line between BTS and MS. In Fig. 4 if angles of arrival at BTSs are measured, the intersection of the two lines giving a position fix for this angle-angle system.

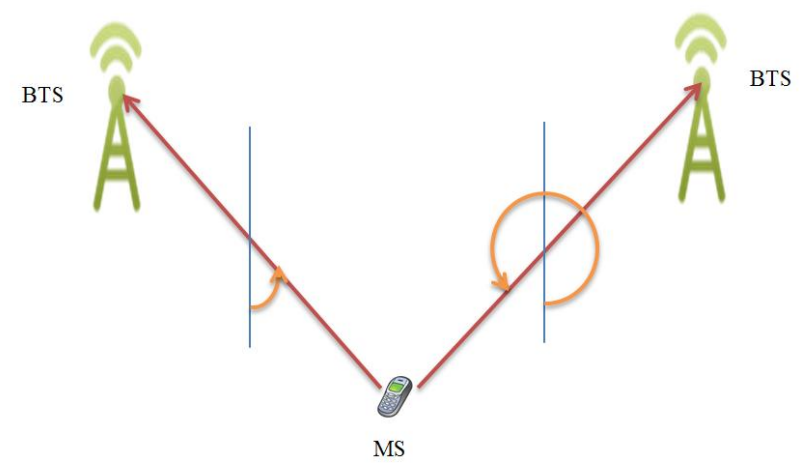

Figure 4. Angle of arrival positioning method

\subsection{Time of Arrival Method (TOA)}

This method is similar to signal strength method for positioning of MS, signal propagation delay from MS to BTSs is measured by additional hardware that is called Location Measurement Unit (LMU) installed in BTS. All LMUs and the MS must share a common clock reference.

\subsection{Time Difference of Arrival Method (TDOA)}

A MS can "listen" to a series of BTSs and measure the time difference between each pair of arrivals. If there are 3 BTS, two independent TDOA measurements can be made. Each TDOA measurement defines a hyperbolic locus. The intersection of two independent TDOA measurements will define the position of MS, as shown in Fig. 5. If instead of MS, each BTS listens to TOA of a MS and sends to a central site, TDOA measurement can be derived from TOA measurement at this central site and the position estimation of MS can be made. The synchronicity of the base stations is the most important issue in this system of positioning. The accuracy of positioning is highly depends on this synchronicity.

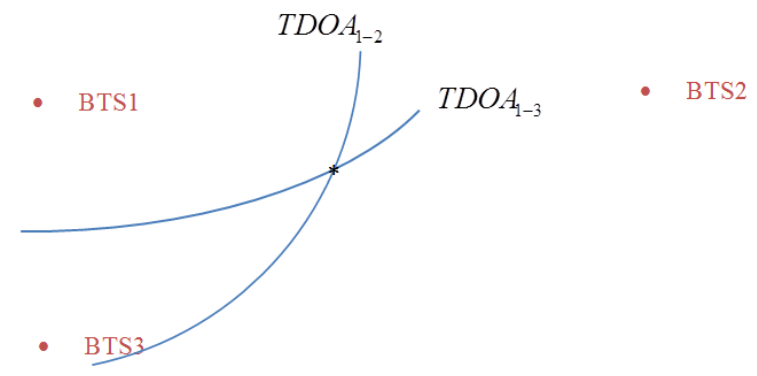

Figure 5. Time Difference of Arrival Method

\section{THE GIS BASED ARCHITECTURE FOR POST- EARTHQUAKE CRISIS MANAGEMENT SYSTEM}

Fig. 6 shows the illustrated GIS based architecture for postearthquake crisis management system. Data base consists of GIS layers, BTS position data and rescue station position data. Server calculates MS position and road finding from each critical point to nearest rescue station.

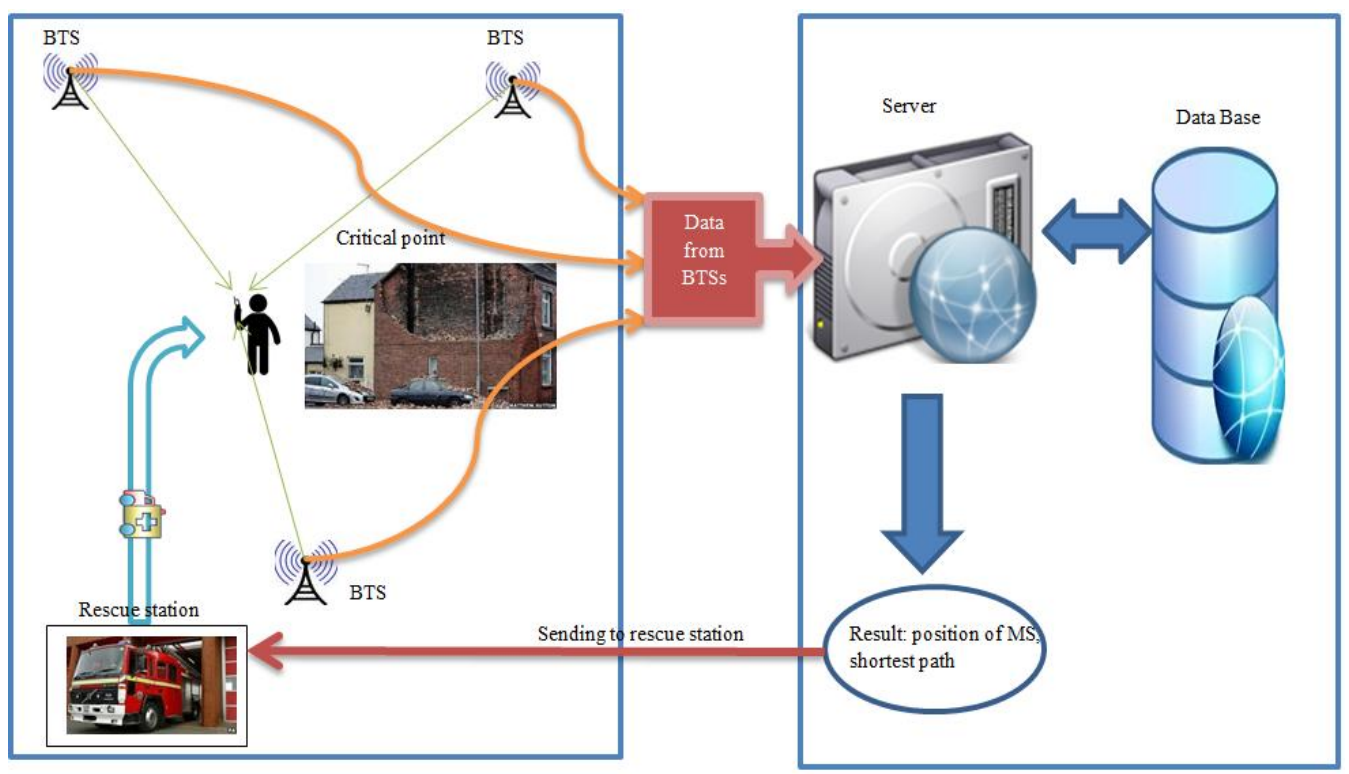

Figure 6. GIS based architecture for post-earthquake crisis management system 


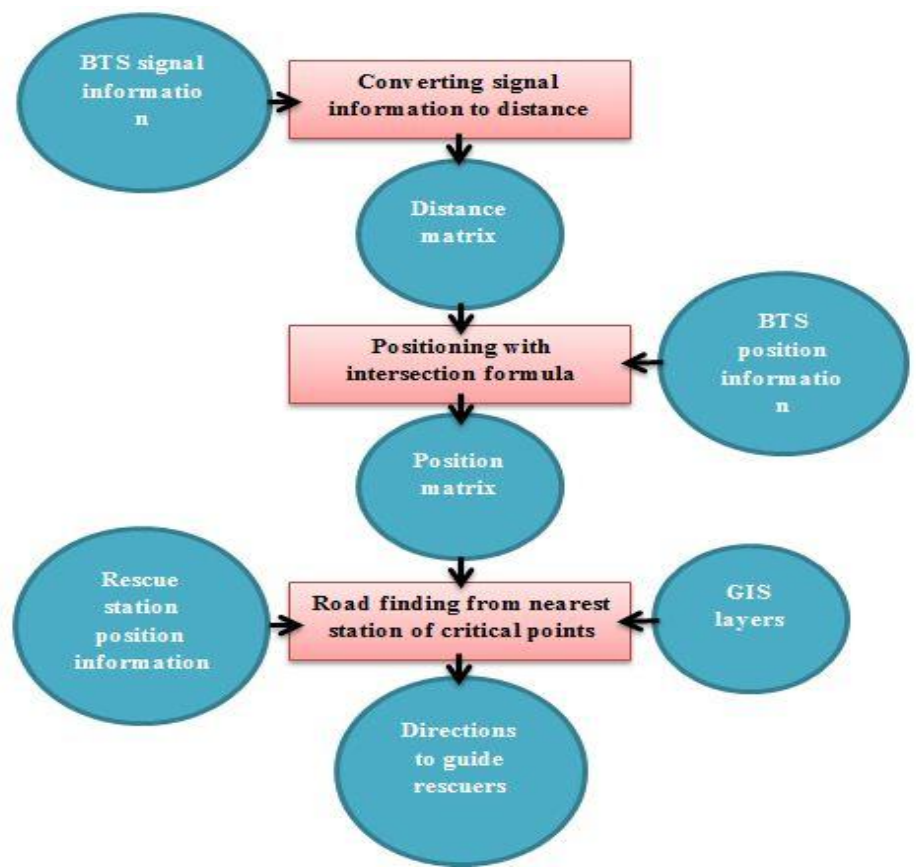

Figure 7. Flowchart of programing

A simulation environment in Matlab software for testing this procedure in a part of district three of Tehran city is done. Fig. 7 shows steps in programing of this procedure. Three critical points, four BTSs and two rescue stations are assumed for this procedure of relief. The positions of critical points are calculated by signal processing and intersection formula (Fig. 8). RMSE of critical points is about 200 meter. Each critical point belonged to a rescue station based on Euclidian (Fig. 9).

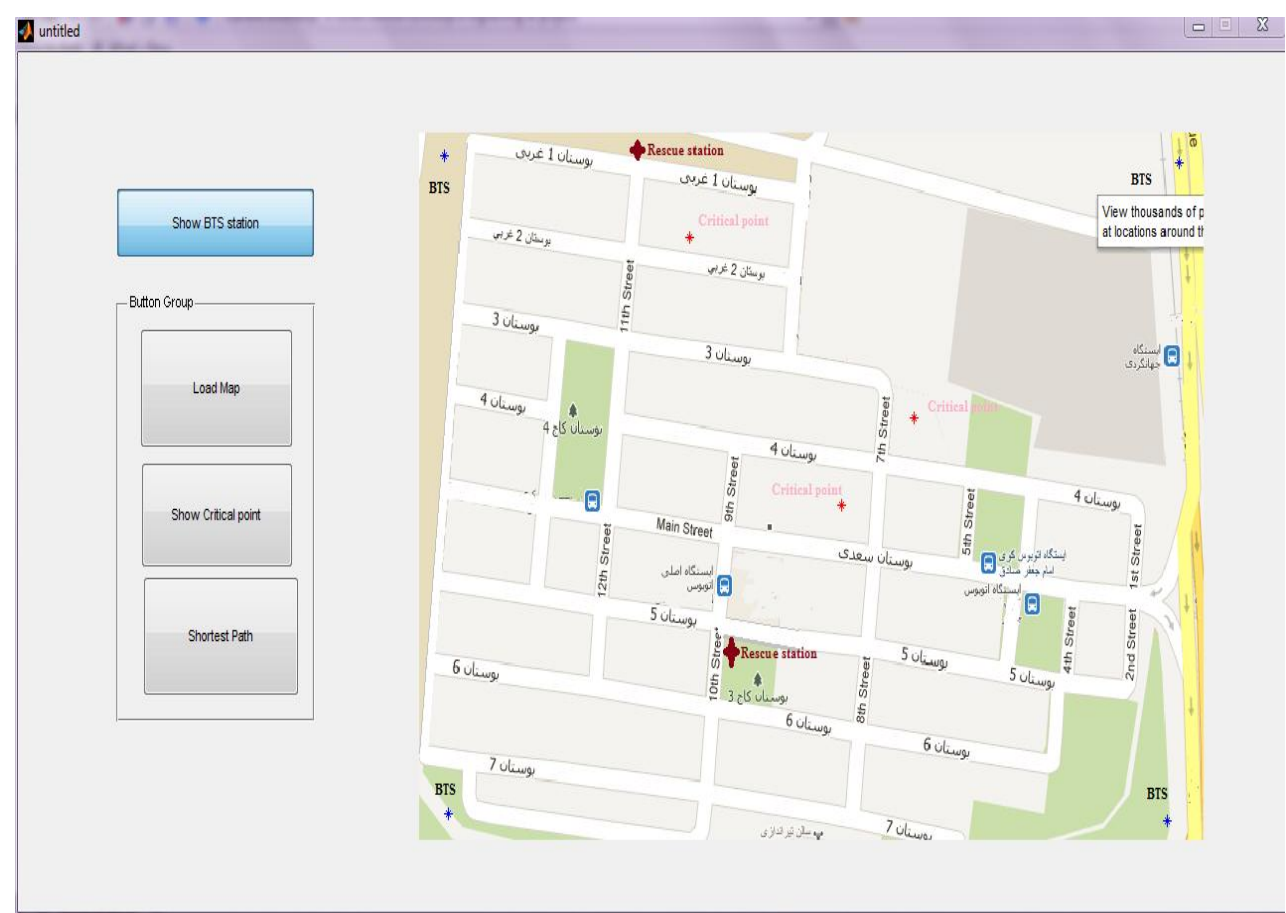

Figure 8. Program interface (critical points, BTSs, rescue stations) 


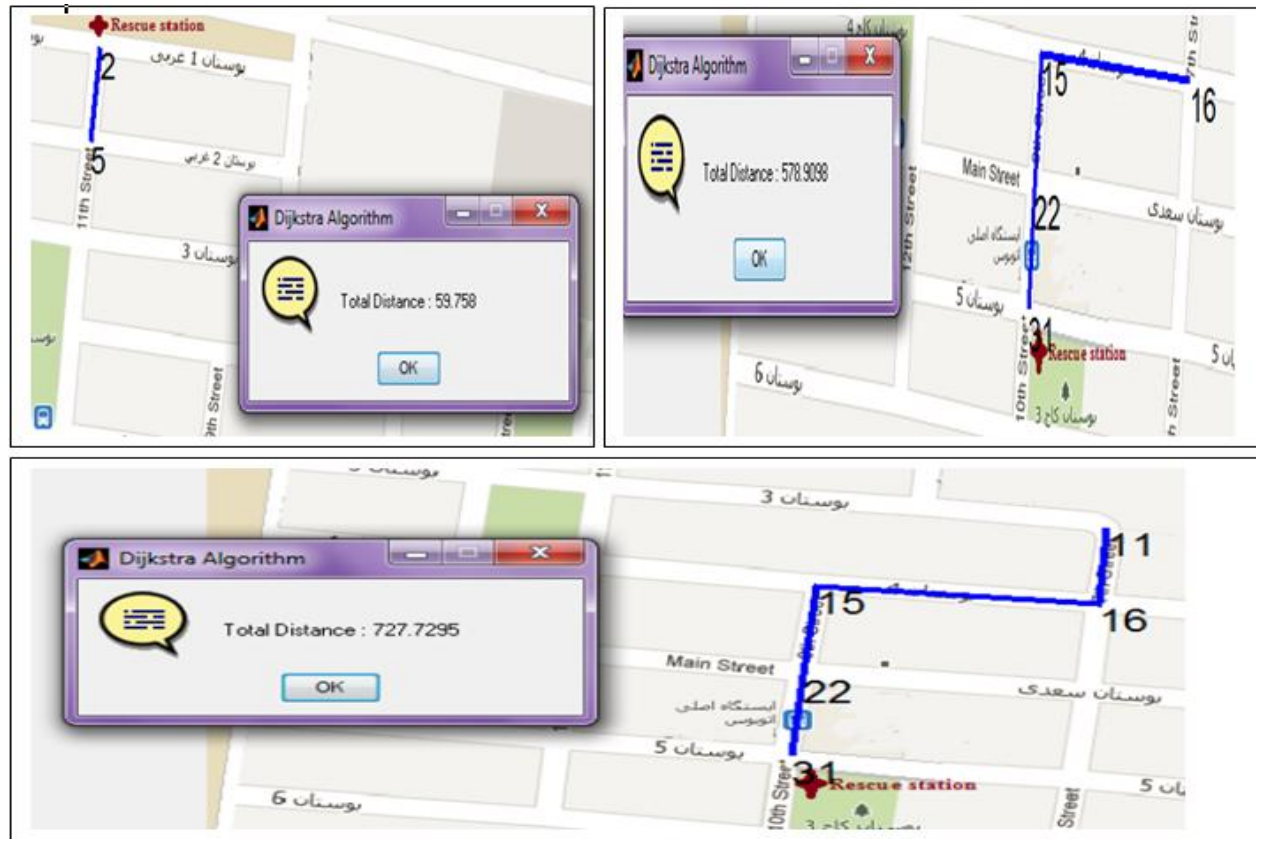

Figure 9. Program interface (Routes)

\section{CONCLUSIONS}

Some positioning method were introduced for cellular network, in this paper. Application and importance of rapid positioning stated for quick response after earthquake and then the proposed GIS based architecture for post-earthquake crisis management system was expressed. The results showed that precision of this method for positioning is appropriate for relief, response times dramatically decreased and this system can be useful for post-earthquake management.

A combination of two or more positioning methods can mitigate the estimation error; improve the availability and accuracy as well as the response time. A future plan is synthesis of positioning methods to improve the accuracy and efficiency.

\section{REFERENCES}

B. Al-Ifan, N. Hamid, M. Othman, S. Subramanian, 2009. A Hybrid Algorithm for Location Mobile Station in Cellular Network. In: International Conference of Future Computer and Communication (ICFCC).

E. Doukhnitch, M. Salamah, 2008. General approach to simple algorithms for 2-D positioning techniques in cellular networks. Computer Engineering Department, Eastern Mediterranean University, Famagusta, TRNC-Mersin 10, Turkey, Received 23 April 2007; received in revised form 1 February 2008; accepted 9 February 2008 Available online 19 February.

G. Kbar, W. Mansoor, 2005. Mobile station location based on hybrid of signal strength and time of arrival, In: IEEE International Conference on Mobile Business, pp. 585 - 591.

R.T. Juang, D. B. Lin, H.P. Lin, 2006. Hybrid SADOA/TDOA Location Estimation Scheme for Wireless Communication Systems. VTC 2006, pp1053-1057.

Samiei, M., Mehrjoo, M. Pirzade, B, 2010. Advances of Positioning Methods in Cellular Networks.
Wan Bejuri, Wan Mohd. Yaakob1, Mohamad, Mohd. Murtadha1 and Sapri, 2011. Ubiquitous Positioning: A Taxonomy for Location Determination on Mobile Navigation System, Faculty of Computer Science \& Information Systems. 